\title{
Current concepts in the management of primary hyperparathyroidism
}

\author{
N Gopalakrishna Iyer · Ashok R. Shaha
}

Received: 5 December 2009

Accepted: 5 February 2010

(C) Indian Association of Surgical

Oncology 2010

N Gopalakrishna Iyer .

Ashok R. Shaha $(\square)$

Head and Neck Service, Memorial

Sloan-Kettering Cancer Center,

New York

e-mail: shahaa@mskcc.org

\begin{abstract}
Primary hyperparathyroidism is the commonest cause of hypercalcemia in the ambulatory setting. Widespread use of routine laboratory screening has resulted in a large number of patients presenting with subclinical disease. In truly asymptomatic patients, consensus guidelines have been developed to determine which patients need definitive treatment. The most common pathologic finding is parathyroid adenoma, followed by hyperplasia, double adenomas and parathyroid carcinoma. The mainstay of treatment is surgery. While there is still an important role for four gland exploration and evaluation, there is now considerable interest in a more focused surgical approach. This paradigm shift is based on localizing studies that combine sestamibi scanning with anatomic imaging, most commonly ultrasound scanning. A range of minimally invasive approaches have been developed to treat parathyroid adenomas, including unilateral and single gland explorations as well as a number of different endoscopic techniques. Intra-operative rapid parathormone assay has replaced histologic examination as a more effective method to confirm the adequacy of surgery in most cases. Functional localization and exploration using a gamma probe has also been described. The management of patients with persistent or recurrent hyperparathyroidism is difficult and requires a multidisciplinary approach.
\end{abstract}

Keywords Sestamibi $\cdot$ intra-operative $\cdot \mathrm{PTH} \cdot$ hypercalcemia

\section{Introduction}

In the mid-80's John Doppman made statement that reflected the expert opinion of his time: 'The only localizing study required in surgery of primary hyperparathyroidism is to localize an experienced parathyroid surgeon.' Since that time, however, there has been a major paradigm shift in the management of primary hyperparathyroidism. The last 20 years have seen advances in localizing techniques such as sestamibi scanning, and the availability of rapid intra-operative PTH assay. Coupled with these, there has been increasing interest in minimally invasive surgical techniques over the classical four-gland exploration, as the procedure of choice in the vast majority of patients with primary hyperparathyroidism, where the enlarged gland has been localize preoperatively. As a result, the endocrine surgeon can no longer be dogmatic about his surgical approach, and needs to be well-trained in minimally invasive procedures as well as four-gland exploration depending on the clinical scenarios, and understand the nuances of current concepts in managing this disease. In this review, we will discuss the approach to primary hyperparathyroidism with an emphasis on the technologies available to the endocrine surgeon. 
Primary hyperparathyroidism results from autonomous secretion of parathyroid hormones from pathologic glands. This results in hypercalcemia, hypophosphatemia and hypercalciuria. Prevalence in the adult population is approximately $1 \%$, and this increases to $2 \%$ in the older population (age $>55$ years), with a male:female ratio of $2-3: 1{ }^{1}$ The most common presentation is sub-clinical disease with elevated calcium detected during routine investigations. ${ }^{2}$ Further questioning may reveal mild symptoms not readily attributed to hypercalcemia. ${ }^{3}$ Classic symptoms of urolithiasis, bone pain, and psychiatric disturbances are less common. The initial aim of managing these patients is to confirm the diagnosis and exclude other causes of hypercalcemia (Table 1), identify and localize the cause for primary hyperparathyroidism and plan the appropriate treatment.

Surgery is the mainstay of treatment for primary hyperparathyroidism. Previously, the standard treatment for all patients was a bilateral neck exploration with identification and evaluation of all four parathyroid glandsthe four-gland exploration. Direct intraoperative examination by the surgeon with occasional histologic examination of frozen sections, allowed experienced endocrine surgeons to treat this condition with a success rate of over $95 \%{ }^{2}$ While this procedure still has a role to play in certain specific clinical scenarios, the current surgical approach is more focused to the pathologic gland based on pre-operative localization. Combined with intraoperative PTH levels to confirm the success of this operation, this focused strategy has a success rate similar to that offered by four-gland exploration. The major caveat to the more focused approach is that they should only be employed in centers with the expertise to perform preoperative localization, and by surgeons experienced in parathyroid surgery.

\section{Surgical anatomy}

Effective parathyroid surgery requires a clear understanding of parathyroid development and surgical anatomy. Parathyroid glands appear as tan to reddish-brown soft, pliable nodules. ${ }^{4,5}$ They are usually ovoid or bean shaped,

Table 1. Causes of hypercalcemia

- Primary hyperparathyroidism

- Malignant disease (metastatic cancer, abnormal production of PTH-related protein, multiple myeloma)

- Pagets disease

- Sarcoidosis

- Thiazide diuretics

- Milk-alkali syndrome

- Benign familial hypocalciuric hypercalcemia

- Pseudohyperparathyroidism

- Hypervitaminosis D but can be elongated or multilobed. Normal glands measure between 3 to $5 \mathrm{~mm}$ and weigh $30-35 \mathrm{mg}$. Most people have four parathyroid glands, although about $10 \%$ have between 5 to 7 glands, while only $2-3 \%$ have fewer than four. The parathyroid glands develop during the sixth week of gestation. The superior glands develop from the fourth branchial pouch, while the inferior glands develop from the third branchial pouch together with the thymus. They then descend to occupy their adult positions in the neck. There are two important consequences to this development. First, the variability in parathyroid gland position usually results from maldescent along the line of descent from the lateral border of the hyoid downwards, adjacent to the carotid sheath. Second, the position of the inferior parathyroid gland is more variable as it traverses a longer distance during descent. The most common locations of the superior and inferior glands are indicated in Table 2. As shown, the superior gland has a more consistent location usually in the vicinity of the superior thyroid pole, except when it is enlarged and pushed back to the para- and retro-esophageal direction, and then into the posterior mediastinum. Occasionally, the superior gland may fail to descend and lie adjacent to the hyoid or parapharyngeal musculature. In contrast, the position of the inferior glands can be quite variable as shown in Table 2 . They are usually found in the vicinity of the inferior thyroid pole, anterior to the recurrent laryngeal nerve. However, these can descend into the mediastinum together with the thymus. The latter is an important point to note in reoperative parathyroid sur-

Table 2. Anatomic location of parathyroid gland (with percentage likelihood). ${ }^{4,5}$

Superior parathyroid gland

- Area of $2 \mathrm{~cm}$ diameter, $1 \mathrm{~cm}$ above intersection of recurrent laryngeal nerve and superior branch of inferior thyroid artery $(80 \%)$

- Behind superior thyroid pole (12\%)

- Tracheo-esophageal groove behind inferior thyroid artery or tubercle of Zuckerkandl

- Above superior thyroid pole

- Retro-esophageal or retro-pharyngeal

- Carotid sheath

- Within thyroid gland

Inferior parathyroid gland

- In the vicinity of the lower thyroid pole (inferior, posterior or lateral) $(61 \%)$

- Thyro-thymic tract and thymic horn (26\%)

- Higher up on the thyroid gland or anterior to the lower thyroid pole (17\%)

- Retromanubrial space

- Superior mediastinum

- Tracheo-esophageal groove

Within thyroid gland 
gery, or in situations where all parathyroid tissue within the thymus need to be removed (e.g. secondary or tertiary hyperparathyroidism or the MEN syndromes) or sternal split may be required. Rarely, parathyroid gland may be intrathyroidal in location. Gland position is symmetrical in $80 \%$ of individuals. The parathyroid surgeon should be aware of the variability in location of the glands. This knowledge is invaluable in situations where pre-operative localization has failed or in reoperative parathyroid surgery. ${ }^{6}$

\section{Pathophysiology}

The main function of the parathyroid glands is calcium homeostasis, through the secretion of parathyroid hormone (PTH). This is an 84 amino acid protein that functions to control calcium levels through its effect on the bone, kidneys and activation of vitamin $\mathrm{D}$. It has a short half-life of only two minutes, which is the reason why intra-operative PTH level monitoring is effective in predicting the successful removal of any pathologic gland. Primary hyperparathyroidism occurs when there is autonomous secretion of PTH from one or more abnormal glands. In contrast, secondary and tertiary hyperparathyroidism occur secondary to chronic renal failure and the resultant hypocalcemia. There are a number of causes for PTH hypersecretion: approximately $85 \%$ of patients with primary hyperparathyroidism have a single parathyroid adenoma, $10 \%$ have four-gland hyperplasia, and $4 \%$ have double adenomas. Fewer than one percent of primary hyperparathyroidism is due to parathyroid carcinoma. Histologic differentiation between adenoma, hyperplasia and carcinoma may be difficult, even more so during frozen section examination. All of these display the same architecture: increased density of chief cells with replacement of normal parathyroid fat. Suffice to say that successful treatment requires identification and removal of all pathologically involved glands.

\section{Clinical assessment}

Few patients present with the classic symptoms of hyperclacemia: 'stones, bones, abdominal groans and psychic overtones'. With the advent of multichannel chemistry, a large proportion of patients present with sub-clinical disease and a laboratory report of hypercalcemia found during routine health screens or investigations for other disorders. $^{1-3}$ Primary hyperparathyroidism is the commonest cause of hypercalcemia in the ambulatory setting. However, it is important to exclude other causes (Table 1). In this respect, the PTH levels are almost diagnosticprimary hyperparathyroidism results in hypercalcemia associated with elevated or inappropriate (high) normal PTH levels. Patients with primary hyperparathyroidism also have low serum phosphorus and high urinary excretion of calcium. The latter is an important contradistinction to benign familial hypocalciuric hypercalcemia $(\mathrm{BFHH})$, which is a rare but often confusing differential diagnosis as PTH levels can also be elevated. Elevated calcium and PTH levels may also be seen in patients taking lithium or thiazide diuretics. Paraneoplastic hypercalcemia secondary to tumor production of parathyroid hormone-related protein (PTH-rp) can usually be differentiated form primary hyperparathyroidism, as this protein (PTH-rp) is not usually detected by the commonly available PTH assays.

The decision to treat patients with primary hyperparathyroidism can be controversial. A suggested algorithm is shown in Fig. 1. Obviously, symptomatic patients need to undergo surgery to remove the pathologically abnormal gland(s). However, a significant proportion of patients are diagnosed with subclinical disease, with an incidental finding of hypercalcemia and high PTH levels. ${ }^{7}$ There is much debate as to whether these patients are truly asymptomatic, as a number of publications have suggested these patients have mild symptoms that resolve post-surgery. ${ }^{3}$ Nonetheless, there still remains a group of patients who are asymptomatic. In 2002, a National Institute of Health (NIH) workgroup produced a set of criteria to determine which patients with asymptomatic primary hyperparathyroidism should undergo surgery (Table 3 ). ${ }^{8}$ Patients who

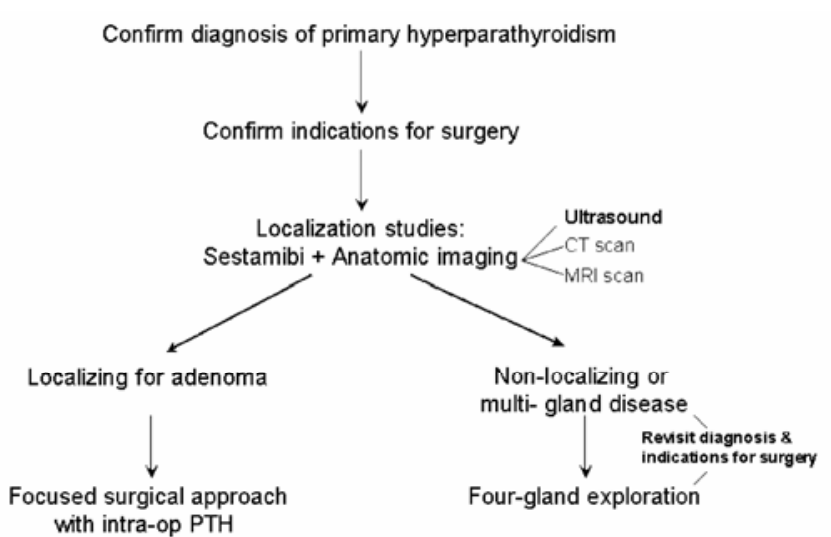

Fig. 1 Approach to primary hyperparathyroidism.

Table 3. Consensus guidelines-criteria for parathyroid surgery in asymptomatic primary hyperparathyroidism ${ }^{8}$

\begin{tabular}{ll}
\hline Age & $<50$ years \\
Serum calcium & $1 \mathrm{mg} / \mathrm{dL}$ above upper limit of \\
& normal \\
24 h urine calcium & $>400 \mathrm{mg}$ \\
Reduction in creatinine & $>30 \%$ \\
$\quad$ clearance & \\
Bone mineral density & $t<-2.5$ at any site \\
\hline
\end{tabular}


do have mild or no symptoms, and do not fit into any of the consensus criteria may be observed with regular follow-up with serum calcium levels every six months, and annual creatinine clearance and dual-energy X-ray absorptiometry testing or bone densitometry scan. The rationale for watchful waiting has also been challenged by focused surgical approaches, which have less morbidity and can even be performed under local anesthesia.

\section{Imaging}

The aim of pre-operative imaging is to localize the offending gland. These investigations are most effective when dealing with parathyroid adenomas, which comprise $85 \%$ of cases. There are a number of different modalities available, listed in Table 4 . The most commonly used are sestamibi nuclear localizing, which is a functional assay and ultrasound scans, which are anatomic. These investigations are critical when planning focused parathyroid exploration or in re-operative parathyroid surgery. Most surgeons would not perform these studies if they are planning a four-gland exploration $a$ priori in a virgin neck. Invasive localizing techniques listed in Table 4 are rarely used due to the high success rates of non-invasive imaging modalities.

\section{Functional imaging}

Sestamibi scanning has become the radiopharmaceutical of choice for nuclear localizing studies, ever since its utility was discovered serendipitously in $1989 .{ }^{9}$ This technique takes advantage of the fact that abnormal parathyroid

Table 4. Parathyroid localizing studies

\begin{tabular}{ll}
\hline Non-invasive studies & \multicolumn{1}{c}{ Invasive studies } \\
\hline - Sestamibi scan & - Angiography \\
- Ultrasound & - Selective venous catheterization \\
- CT scan & - Ultrasound-guided needle biopsy/sampling \\
- MRI scan & \\
- SPECT scan & \\
- PET scan & \\
\hline
\end{tabular}

Table 5. Causes of failed parathyroid surgery

\footnotetext{
- Incorrect diagnosis

- Inexperienced surgeon

- Undetected enlarged gland

- Multiple-gland disease

- Failure to locate an ectopic gland

- Supernumerary gland

- Parathyroid carcinoma

- Parathyromatosis from spillage
}

glands have more mitochondria, hence tend to sequester sestamibi. ${ }^{10}$ There are several different techniques used in practice, the most common is a double-phase scan: sestamibi is administered intravenously, and images are taken early (10 and $30 \mathrm{~min}$ ) and late (at $2 \mathrm{~h}$ ). This reveals a differential washout between the thyroid and the parathyroid gland, as the latter retains the radiopharmaceutical, allowing a delayed image of the gland. The addition of CT scanning to this technique provides threedimensional localization, and is referred to as the sestamibi single photon emission computed tomography (SPECT). ${ }^{11}$ While SPECT is usually unnecessary in most cases of primary hyperparathyroidism, it may be invaluable in locating a previously missed parathyroid gland in the neck or superior mediastinum, or when there are thyroid nodules that obscure the parathyroid lesion. A less commonly used technique is to use sestamibi with radioactive iodine or technetium, and to perform subtraction imaging to distinguish the parathyroid gland from thyroid nodules. However, this is not usually necessary unless the thyroid glands reveal clinically obvious abnormalities.

Regardless of the technique used, sestamibi scanning as a single modality for identifying parathyroid adenomas, has a reported sensitivity of $54-100 \%$, with most recent series in the $80-90 \%$ range. ${ }^{2,12,13}$

\section{Anatomic imaging}

Ideally, localizing techniques combine functional imaging with sestamibi scanning with anatomic imaging, either ultrasound, CT or MRI. The most frequently used anatomic modality is ultrasound scans. Ultrasound has the advantage of being cheap, does not expose the patient to ionizing radiation, and can easily be performed as an office procedure. It is increasingly being performed by the endocrine surgeons themselves, and in many situations, can be performed pre-operatively to direct exploration prior to placing the incision. Normal parathyroids are usually not seen on ultrasound. However, parathyroid adenomas usually have a typical sonographic appearance: round to ovoid, measuring $8-20 \mathrm{~mm}$, homogenous and hypoechoic compared to the thyroid gland and often vascular on Doppler flow. The main disadvantage of the ultrasound is that it is user-dependent and does not image the retro-manubrial and mediastinal glands. As a single modality for identification of parathyroid adenoma, ultrasound has a sensitivity of $27-89 \%$. This increases to $78-$ $96 \%$ if ultrasound is combined with sestamibi scanning, which is highly accurate in primary hyperparathyroidism. $^{2,13}$

Several recent reports have examined the role of SPECT scans in localizing parathyroid adenomas in patients who have not been previously treated. Results have been variable: some studies have shown increased sensitivities and positive predictive values while others have reported lim- 
ited clinical value in this scenario. ${ }^{14}$ SPECT scans may be more useful in localization for patients who have failed previous surgery and present with persistent primary hyperparathyroidism, and reoperative parathyroid surgery has been planned.

\section{Surgical management}

The mainstay of treatment for primary hyperparathyroidism is surgery. The decision to undertake four-gland exploration versus a more focused approach depends on effective pre-operative localization, the primary diagnosis (adenoma versus hyperplasia) and the ability of the surgeon in performing either procedure (Figure 1). Regardless of the procedure planned, patients should be adequately counseled on the risks of surgery. These include major complications (hematoma, recurrent laryngeal nerve injury, hypocalcemia etc), the chances of converting from a focused approach to four-gland exploration, where applicable, and the possibility that surgery may be unsuccessful in some unpredictable (albeit uncommon) circumstances. In most cases, surgery is performed under general anesthesia. However, focused techniques can be performed using monitored anesthetic care with local or regional anesthetic blocks. This may be useful in high risk patients not-suitable for general anesthesia.

\section{Four-gland exploration}

Twenty years ago. four-gland exploration was the gold standard procedure for patients with primary hyperparathyroidism. In experienced hands, this has a success rate of over $95 \%$. $^{2}$ In centers where there are no facilities for pre-operative localization, this still remains the procedure of choice. Otherwise, it is limited to a few specific situations: failure to pre-operatively localize a parathyroid adenoma, presumptive diagnosis of multi-gland disease (hyperplasia or multiple adenomas) and re-operative parathyroid surgery, where the primary procedure has failed. It is an important technique for all endocrine surgeons to be familiar with, as occasionally, focused techniques may fail to identify an offending gland, and the operation needs to be extended to a formal bilateral exploration. Alternatively, intra-operative PTH levels may fail to appreciably drop post-resection of an abnormal parathyroid gland, necessitating exploration of the remaining glands. Patients should also be made aware of this possibility prior to any focused approach.

The usual technique involves making a $4-6 \mathrm{~cm}$ transverse incision in the neck similar to a thyroidectomy incision. Four-gland exploration involves mobilizing both lobes of the thyroid, identification of both recurrent laryngeal nerves and the neck is explored to identify and evaluate all four parathyroid glands. There are some variations in techniques used. Some surgeons routinely take-down both superior poles and some send tissue from all four parathyroid glands for frozen section histological examination to confirm the tissue type. Search for the parathyroid glands are performed systematically on each side, with a clear understanding on the usual sites where they may be (Table 2). If the diagnosis is a parathyroid adenoma, the offending gland is removed and the remaining three glands are left untouched. Similarly, for double adenomas, only the abnormal glands are removed and the normal ones are left intact. Four-gland hyperplasia is either treated with subtotal parathyroidectomy- removing three and a half glands, leaving half in its native location with its vascular pedicle, or all four glands are excised, with some parathyroid tissue implanted either in the sternocleidomastoid muscle or in the forearm.

\section{Focused parathyroidectomies}

As more than $85 \%$ of patients with primary hyperparathyroidism have a parathyroid adenoma, removal of the single, offending gland is the only required procedure. ${ }^{15}$ There are two important pre-requisites for performing focused parathyroidectomies: first, pre-operative localization has to successfully identify the offending gland, and second, there should be a way after removal of this gland to predict that surgery would be curative. ${ }^{16,17}$ As stated before, combining sestamibi scans with anatomic imaging has a high sensitivity in localizing parathyroid adenoma. Once identified, these can be removed through a more focused approach. This can be a unilateral neck exploration or a single-gland exploration. The former entails making a small, $3-5 \mathrm{~cm}$ incision and exploring one side of the neck to identify both parathyroid glands and the recurrent laryngeal nerve. A focused single-gland exploration can be performed through a smaller incision $(2-3 \mathrm{~cm})$ placed over the putative location of the abnormal gland. A lateral trapdoor approach, in a plane between the lateral edge of the strap muscles and the sternocleidomastoid, allows easy access to the thyroid and parathyroid glands. Some surgeons do not actively identify the recurrent laryngeal nerve, but limit their dissection to the immediate environs of the abnormal gland.

Confirmation that this procedure was successful is critical and avoids further exploration and future recurrence. Rapid intra-operative measurement of PTH levels would confirm this notion. PTH levels are usually measured at the start of surgery and 10 min after removal of the gland. The assay usually takes between 15-20 min to perform and many high-volume centers have the necessary equipment in the operating suite. A drop in PTH of more than $50 \%$ of the pre-resection measurement indicates that the source of the hyperparathyroidism has been removed. It is also recommended that the postoperative PTH levels be in the normal range. An analysis of 210 published papers which used intra-operative PTH assays, 
showed that unilateral exploration was successful in $94.5 \%$ of cases studied. ${ }^{18}$ The remaining $5.5 \%$ required conversion to four-gland exploration. Surgical failure was seen in $1.3 \%$ of cases, where persistent hypercalcemia was seen post-surgically. Frozen section histology has limited role in these situations. Apart from confirming that the tissue removed is parathyroid tissue, frozen section examination is usually unable to distinguish between parathyroid adenomas and hyperplasia. The only information usually gained is to weigh the specimen and confirm that the specimen is indeed parathyroid tissue.

\section{Minimally invasive approaches}

Although there has been much interest in endoscopic parathyroidectomy techniques, it is sobering to note that single-gland exploration can often be performed with a $2 \mathrm{~cm}$ neck incision over a well-localized adenoma. Nonetheless these are being increasingly preformed in highvolume centers around the world. A number of different endoscopic techniques have been described. One involves the use of gas insufflation, which allows incisions to be placed at a distance from the gland, either infra-clavicular or even in the axillae. ${ }^{19}$ Alternatively, a small lateral neck incision can be made to introduce an endoscope, and dissection performed while an assistant directs the scope and retracts with small instruments. ${ }^{20}$ Bilateral explorations have also been performed using small midline incisions. ${ }^{21}$ The technique is similar to video assisted thyroidectomies, and has been unimaginatively called minimallyinvasive video-assisted parathyroidectomies. Regardless of technique, patients should have pre-operative localization to confirm the diagnosis, and have an anatomy favorable for this approach (i.e. non-obese, no thyroid nodules and no short neck). ${ }^{20}$ In patients with skin creases in the neck, the cosmetic difference between these and focused techniques are often minimal. Radioguided surgery is a variation on minimally invasive techniques that directs the surgeon to the abnormal parathyroid gland. ${ }^{22}$ Patients are given $99 \mathrm{mTc}$-sestamibi on the day of surgery, and undergo imaging to confirm the location (and mark) the location of the gland. A small incision is placed over the putative site, and a gamma camera is used to direct the surgeon to the abnormal gland, in a similar technique to sentinel node biopsy. Radioactivity of more than $20 \%$ of background activity is essentially a confirmation that the tissue removed is parathyroid adenoma and indicates successful surgery. This technique has not gained widespread popularity, yet continues to be used in several centers.

\section{Parathyroid re-exploration}

Regardless of the surgical approach to primary hyperparathyroidism, no treatment is $100 \%$ curative. Patients with persistent or recurrent hyperparathyroidism after previous surgery are difficult to treat. ${ }^{6}$ The best strategy is to prevent the need for re-operative surgery in the first instance. Notwithstanding the technical difficulties in dealing with a hostile neck, the surgeon has to contend with a frustrated patient with high expectations of success. Causes of a failed initial operation are listed in Table 4, and it is incumbent on the treating surgeon to review previous surgical and pathologic findings, if possible, directly engage the previous surgeon and discuss his approach and findings. This may provide important clues as to the reason for a failed initial operation. A suggested algorithm is shown in Fig. 2. Patients should undergo a full re-evaluation including confirmation of the diagnosis (and exclusion of differential diagnoses for hypercalcemia), reassessment as to the indication for surgery and repeat localization. Localization should be a combination of functional and anatomic imaging, and this requires a combination of sestamibi and CT scans or MRI, or even SPECT scans. Particular attention should

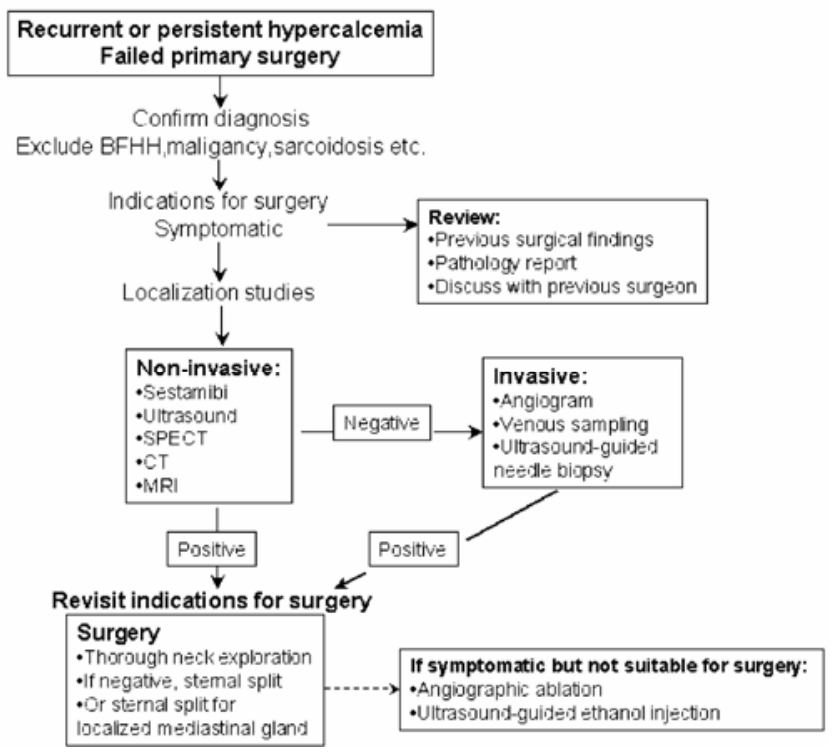

Fig. 2 Approach to parathyroid re-exploration.

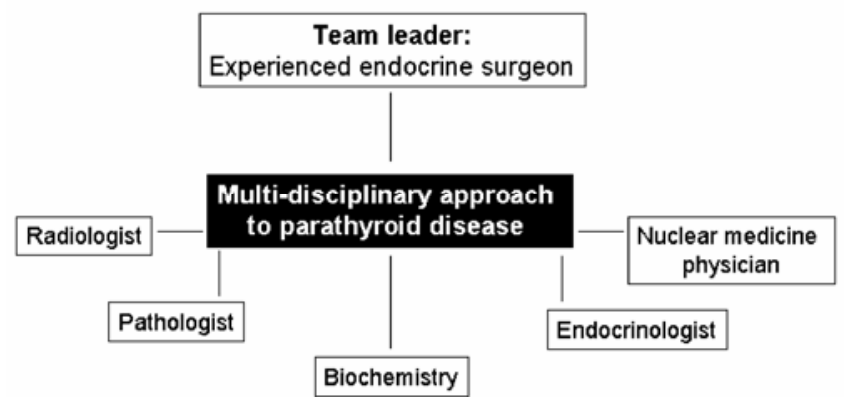

Fig. 3 Multidisciplinary approach to management of primary hyperparathyroidism. 
be paid to imaging the mediastinum to identify ectopic or supernumerary glands. Successful managament of patients with primary hyperparathyroidism and a failed operation, requires a multidisciplinary approach (Fig. 3). The parathyroid surgeon as the team leader, needs to engage the endocrinologist, nuclear medicine physician, radiologist and pathologist along with the biochemistry laboratory.

\section{Parathyroid carcinoma}

Parathyroid carcinoma is a rare cause of primary hyperparathyroidism, and fewer than 1000 cases have been reported in the literature. ${ }^{23,24}$ As such, data for treatment modalities and outcome are limited. The main preoperative finding is high calcium and PTH levels, and patients are often symptomatic and may develop hypercalcemic crisis. Nuclear imaging may localize the lesion but are not useful for diagnosis. The presence of metastatic disease to regional nodes or distant sites may confirm the diagnosis prior to surgery, The diagnosis can also be made intra-operatively on the basis of tumor invasion of adjacent structures. Frozen section examination is usually unreliable, and the diagnosis may only be confirmed after routine histological examination. Micro- scopic features suggesting malignancy include a high mitotic rate, and capsular, vascular or neural invasion. Treatment usually entails removal of the tumor, usually with adjacent structures, which are invaded that includes the ipsilateral thyroid lobe, and overlying strap muscles if applicable. The role of central compartment nodal clearance is undetermined, but obviously enlarged nodes should be removed with the primary tumor.

\section{Conclusions}

The treatment of primary hyperparathyroidism has undergone many paradigm shifts in the last two decades. Surgery remains the mainstay of treatment, but the endocrine armamentarium available to the surgeon has increased considerably. The role of preoperative localization using sestamibi scanning and intra-operative PTH assays cannot be overstated. Successful treatment requires that the surgeon is not only skilled in surgical techniques but has a clear understanding of the tools at his disposal. Engaging a multidisciplinary team is crucial in difficult circumstances, especially when dealing with persistent or recurrent disease. It is therefore incumbent on the surgeon to remain current with the available literature on the subject and constantly engage colleagues in all fields.

\section{References}

1. Bilezikian JP, Silverberg SJ. Clinical practice. Asymptomatic primary hyperparathyroidism. N Engl J Med. 2004;350:1746-1751

2. McHenry CR. What's new in general surgery: endocrine surgery. J Am Coll Surg. 2002; 195: 364-371

3. Pasieka JL. What's new in general surgery: endocrine surgery. J Am Coll Surg. 2004;199:437-445.

4. Hunt PS, Poole M, Reeve TS. A reappraisal of the surgical anatomy of the thyroid and parathyroid glands. Br J Surg. 1968; 55:63-66

5. Akerstrom G, Malmaeus J, Bergstrom R. Surgical anatomy of human parathyroid glands. Surgery. 1984;95: 14-21

6. Shaha AR. Parathyroid re-exploration. Otolaryngol Clin North Am. 2004;37: 833-843, x.

7. Silverberg SJ, Bilezikian JP. The diagnosis and management of asymptomatic primary hyperparathyroidism. Nat Clin Pract Endocrinol Metab. 2006;2:494-503.

8. Bilezikian JP, Potts Jr. JT, Fuleihan $\mathrm{Gel} \mathrm{H}$ et al. Summary statement from a workshop on asymptomatic primary hyperparathyroidism: a perspective for the 21st century. J Clin Endocrinol Metab. 2002;87:5353-5361.

9. Coakley AJ, Kettle AG, Wells CP et al 99 Tcm sestamibi - a new agent for parathyroid imaging. Nucl Med Commun. 1989; 10: 791-794.

10. Shaha AR. Localization studies for hyperparathyroidism: implications for surgery. Acta Otorhinolaryngol Belg 2001;55:139-145.

11. Buck AK, Nekolla S, Ziegler S et al. Spect/Ct. J Nucl Med. 2008; 49: 1305-1319.

12. Friedman K, Somervell $\mathrm{H}$, Patel $\mathrm{P}$ et al. Effect of calcium channel blockers on the sensitivity of preoperative $99 \mathrm{mTc}-\mathrm{MIBI}$ SPECT for hyperparathyroidism. Surgery. 2004; 136:1199-1204

13. McHenry CR, Lee K, Saadey J et al. Parathyroid localization with technetium-99m-sestamibi: a prospective evaluation. J Am Coll Surg. 1996; 183:25-30

14. Palestro CJ, Tomas MB, Tronco GG. Radionuclide imaging of the para- thyroid glands. Semin Nucl Med. 2005;35:266-276.

15. Carling T, Udelsman R. Focused approach to parathyroidectomy. World J Surg. 2008;32:1512-1517.

16. Shaha AR, Patel SG, Singh $B$. Minimally invasive parathyroidectomy: the role of radio-guided surgery. Laryngoscope. 2002;112:2166-2169.

17. Udelsman R, Donovan PI, Sokoll LJ. One hundred consecutive minimally invasive parathyroid explorations. Ann Surg. 2000;232:331-339.

18. Ruda JM, Hollenbeak CS, Stack Jr BC. A systematic review of the diagnosis and treatment of primary hyperparathyroidism from 1995 to 2003. Otolaryngol Head Neck Surg. 2005; 132:359-372.

19. Ikeda $Y$, Takami $H$, Niimi $M$ et al. Endoscopic thyroidectomy and parathyroidectomy by the axillary approach. A preliminary report. Surg Endosc. 2002;16:92-95.

20. Terris DJ, Stack Jr. BC, Gourin CG. Contemporary parathyroidectomy: exploiting technology. Am J Otolaryngol. 2007;28:408-414. 
21. Miccoli P, Berti P, Materazzi G et al. Endoscopic bilateral neck exploration versus quick intraoperative parathormone assay (qPTHa) during endoscopic parathyroidectomy: A prospective randomized trial. Surg Endosc. 2008;22:398-400.

22. Murphy C, Norman J. The $20 \%$ rule: a simple, instantaneous radioactivity measurement defines cure and allows elimination of frozen sections and hormone assays during parathyroidectomy. Surgery. 1999;126:10231028; discussion 1028-1029.

23. Shaha AR, Shah JP. Parathyroid carcinoma: a diagnostic and therapeutic challenge. Cancer. 1999;86: 378380 .
24. Hundahl SA, Fleming ID, Fremgen AM, Menck HR. Two hundred eighty-six cases of parathyroid carcinoma treated in the U.S. between 1985-1995: a National Cancer Data Base Report. The American College of Surgeons Commission on Cancer and the American Cancer Society. Cancer. 1999;86:538-544. 